\title{
Las Tecnologías de Información y Comunicaciones en la formación del ingeniero industrial
}

\author{
Alba Soraya Aguilar-Jiménez ${ }^{\text {a }}$, Manuel Arturo Jiménez Ramírez \\ ${ }^{a}$ Grupo de Investigación GeeTIC, Facultad de Ingeniería Industrial, Universidad Pontificia \\ Bolivariana Seccional Bucaramanga Colombia. Email: alba.aguilar@upb.edu.co. ${ }^{\text {b}}$ Grupo de \\ Investigación GeeTIC, Facultad de Ingeniería Industrial, Universidad Pontificia Bolivariana \\ Seccional Bucaramanga Colombia. Email:manuel.jimenezr@upb.edu.co
}

\section{Resumen}

La dinámica actual de las Tecnologías de Información y Comunicaciones (TIC) ha sumergido a organizaciones y sociedad en general en un entorno cambiante en donde la facilidad de acceso a la tecnología impone retos importantes, creación de nuevas formas de gestionar los recursos y procesos de negocio, aumentar su eficiencia y efectividad, dinamizar los procesos de toma de decisiones, actuar con mayor rapidez ante las exigencias del mercado y acercar a empresas, competidores y consumidores en un nuevo entorno virtual.

El objetivo del trabajo es presentar un marco conceptual y contextual de la importancia de la inclusión de contenidos asociados a las TIC en la formación profesional del ingeniero industrial, que sirva de base al proceso de transformación curricular en que se encuentran el programa de grado en la Universidad Pontificia Bolivariana seccional Bucaramanga (UPB BGA).

Con el fin de comprender las tendencias en relación a las TIC, e Internet en especial, tanto en los negocios como en diversos aspectos de la vida de las personas, se realizó un análisis del contexto y una búsqueda refinada de publicaciones de los últimos 8 años en la base de datos SCOPUS que evidencia un abanico cada vez más amplio en temas de investigación asociados con las TIC.

De la revisión realizada se identifica un amplio espectro de áreas de trabajo para el programa de Ingeniería Industrial de la UPB BGA, no solo en lo que respecta al contexto regional local que enfrenta retos bien importantes de cara al futuro, sino en el contexto mundial en una industria con tecnologías disruptivas, cambios en los modelos de negocio y de paradigmas, en donde las TIC se constituyen en una condición evidente. Estos cambios implican 
una transformación para la cual el profesional del futuro debe estar preparado.

Palabras claves: TIC, tecnologías emergentes, currículo, formación profesional, Ingeniería Industrial.

\section{Introducción}

Las Instituciones de Educación Superior en Colombia -IES, han venido afrontando una serie de cambios asociados con la transformación digital, que se caracterizan por la facilidad de acceso a la información y comunicación de una sociedad interconectada. Esta situación viene inquietando a las IES, cuyo rol les exige repensar sus procesos formativos y adecuarlos a las necesidades del entorno laboral y social, entorno en el que la tecnología juega un papel relevante

La Universidad Pontificia Bolivariana Seccional Bucaramanga (en adelante UPB BGA), es una entidad educativa con acreditación de Alta Calidad Multicampus (Ministerio de Educación Nacional, 2018), que cuenta con una Sede Central en Medellín y con seccionales en Bucaramanga, Montería y Palmira. Como Institución le apuesta a la calidad de su oferta académica, ofreciendo carreras profesionales y de postgrado competitivas a nivel regional, nacional e internacional. Esta apuesta implica procesos de mejoramiento continuo que incluyen la revisión constante de sus currículos y la transformación enfocada a ofrecer programas pertinentes y de calidad desde la perspectiva académica, científica y con carácter ético y moral propios de su sello Pontificio (Universidad Pontificia Bolivariana, 2016, 2019b).

Uno de los programas que ofrece la UPB BGA es el programa de grado de Ingeniería Industrial $^{1}$, que consta de 10 semestres académicos conformados por cuatro ciclos de formación: Ciclo básico disciplinar, Ciclo de formación humanística, Ciclo profesional (asignaturas troncales obligatorias) y Ciclo de Integración (Asignaturas optativas de profundización), siendo los dos últimos sobre los que recae la formación en la disciplina y que a su vez comprende las líneas de formación en Métodos cuantitativos, Métodos cuantitativos aplicados, Sistemas de gestión y de información. Esta última línea intenta, con dos asignaturas troncales, abordar los elementos del uso de las Tecnologías de Información y Comunicaciones (TIC) en la empresa, pero se enfoca desde conceptos tradicionales que

\footnotetext{
1 El Programa de grado de Ingeniería Industrial en Colombia se puede asimilar al programa de Ingeniería en Organización Industrial de España
} 
pierden vigencia rápidamente dada la dinámica tecnológica (Universidad Pontificia Bolivariana, 2019a).

En el marco del proceso de autoevaluación del programa (Ingeniería Industrial UPB Bucaramanga, 2018), y atendiendo los requerimientos del entorno, se realiza un análisis preliminar que permite conocer la expectativa de empresarios y egresados, en relación al perfil profesional esperado del Ingeniero Industrial. Este análisis permite identificar oportunidades de mejora, tales como la importancia de contar con profesionales con competencias para la resolución de problemas en entornos complejos con múltiples variables y en entornos cambiantes y capaces de integrar conceptos adquiridos a lo largo de la carrera en la solución de problemas reales. Igualmente se evidencia la importancia del pensamiento prospectivo y estratégico para proyectar la organización, mayor capacidad de visualizar tendencias en los sectores de tal forma que, a las empresas, se les proporcione nuevos horizontes para desarrollarse en ese sentido, destacando la importancia de fortalecer la apropiación de conocimiento sobre uso de software especializado para soportar la toma de decisiones, modelos de aprendizaje computacional (inteligencia artificial) y minería de datos.

Dadas las oportunidades de mejora, y partiendo del perfil de egreso del profesional, que contempla la formación en competencias para "concebir, diseñar, implementar y mejorar procesos organizacionales desde una perspectiva sistémica y con criterios tecnológicos y de sostenibilidad, capaz de tomar decisiones con criterios éticos fundamentados en el Humanismo Cristiano, que contemplen a la persona como centro de toda organización y con responsabilidad social y respeto al medio ambiente" (Universidad Pontificia Bolivariana, 2019a), el programa de grado de Ingeniería Industrial ha iniciado un proceso de transformación curricular a partir de un análisis interno y externo en busca de mejorar y enriquecer la oferta académica e incorporar los ajustes necesarios que permitan al futuro profesional ser competente ante la dinámica global en la que se encuentra inmerso. Parte de este trabajo corresponde a la revisión conceptual y contextual de la carrera de Ingeniería Industrial desde la perspectiva internacional, nacional y local en las diferentes áreas del saber, razón por la cual en este documento se pretende realizar un análisis de una de las áreas de interés, el área de las Tecnologías de Información y Comunicaciones, y para ello se presenta un marco conceptual y contextual de la importancia de la inclusión de contenidos asociados a las TIC en la formación profesional del ingeniero industrial, que sirva de base al proceso de transformación curricular en que se encuentran el programa de grado en la UPB BGA. 


\section{Revisión de literatura}

Las Tecnologías de Información y Comunicaciones se pueden definir como el conjunto de elementos hardware, software, datos, telecomunicaciones, incluido Internet (Laudon \& Laudon, 2007), que emplean las organizaciones para la gestión de la información, de modo que los Sistemas de información comprenden las tecnologías, datos y personas involucradas en la gestión de información y servicios de comunicación (Davis, 2000). En otras palabras, los sistemas de información son los fines y las tecnologías de información son los medios (Pérez González, 2005).

La robustez creciente de las tecnologías y el crecimiento exponencial de la información en el universo digital abre posibilidades en un espectro muy amplio para las organizaciones y ya desde hace más de una década, Carr en su libro "IT Doesn’t Matter" argumentaba que las TIC habían llegado a ser un simple factor de producción: un elemento en el proceso de producción, que es necesario para la competitividad pero no suficiente para obtener ventajas (Carr, 2004). La dinámica actual de las Tecnologías de Información y Comunicaciones ha sumergido a organizaciones y sociedad en general en un entorno cambiante en donde la facilidad de acceso a la tecnología impone retos importantes, creación de nuevas formas de gestionar los recursos y procesos de negocio, aumentar su eficiencia y efectividad, dinamizar los procesos de toma de decisiones, actuar con mayor rapidez ante las exigencias del mercado y acercar a empresas, competidores y consumidores en un nuevo entorno virtual (Aguilar-Jimenez, 2010).

Por lo anterior, y en función de dar cumplimiento a este perfil, parece importante estudiar las tendencias en relación a las TIC, e Internet en especial, tanto en los negocios como en diversos aspectos de la vida de las personas. Para ello se inició con una búsqueda refinada de publicaciones de los últimos 8 años en la base de datos SCOPUS, lo que evidencia un abanico cada vez más amplio en temas de investigación asociados con las TIC, y para el caso que nos ocupa, para las TIC en los procesos de negocio en las organizaciones. De este proceso se pudo identificar que la mayoría de los estudios $(38,6 \%)$ se encuentran en las áreas de negocios y gestión seguido por un $18 \%$ en economía y afines y $17 \%$ en áreas de ingeniería y se resalta una tendencia creciente de estudios (10,5\% en 8 años) que publican en Ciencia de las Decisiones basadas en las TIC.

Adicionalmente se resalta que los países que más están publicando en esta área son en su orden Estados Unidos, España y Reino Unido pero la filiación que predomina en los autores es de la Universidad Tecnológica de Malasia seguida por dos universidades importantes de Valencia como son las Universidades Politécnica de Valencia y la Universidad de Valencia. 
Ahora bien, haciendo un análisis de las áreas de interés de las publicaciones entre 2010 y 2018 de temas de TIC para áreas de gestión y negocios, se identifican temáticas clásicas como es el uso de TIC para la gestión estratégica, la cadena de suministro, la innovación, el modelado, el desarrollo industrial de empresas de todos los tamaños, el emprendimiento o la industria de servicios y aparecen tendencias emergentes como la gestión de comunidades virtuales, gestión del conocimiento, toma de decisiones basadas en el análisis de datos, negocio electrónico, los agro-negocios y el almacenamiento en la nube como se puede apreciar en el diagrama de burbujas que aparece en la Fig. 1. 


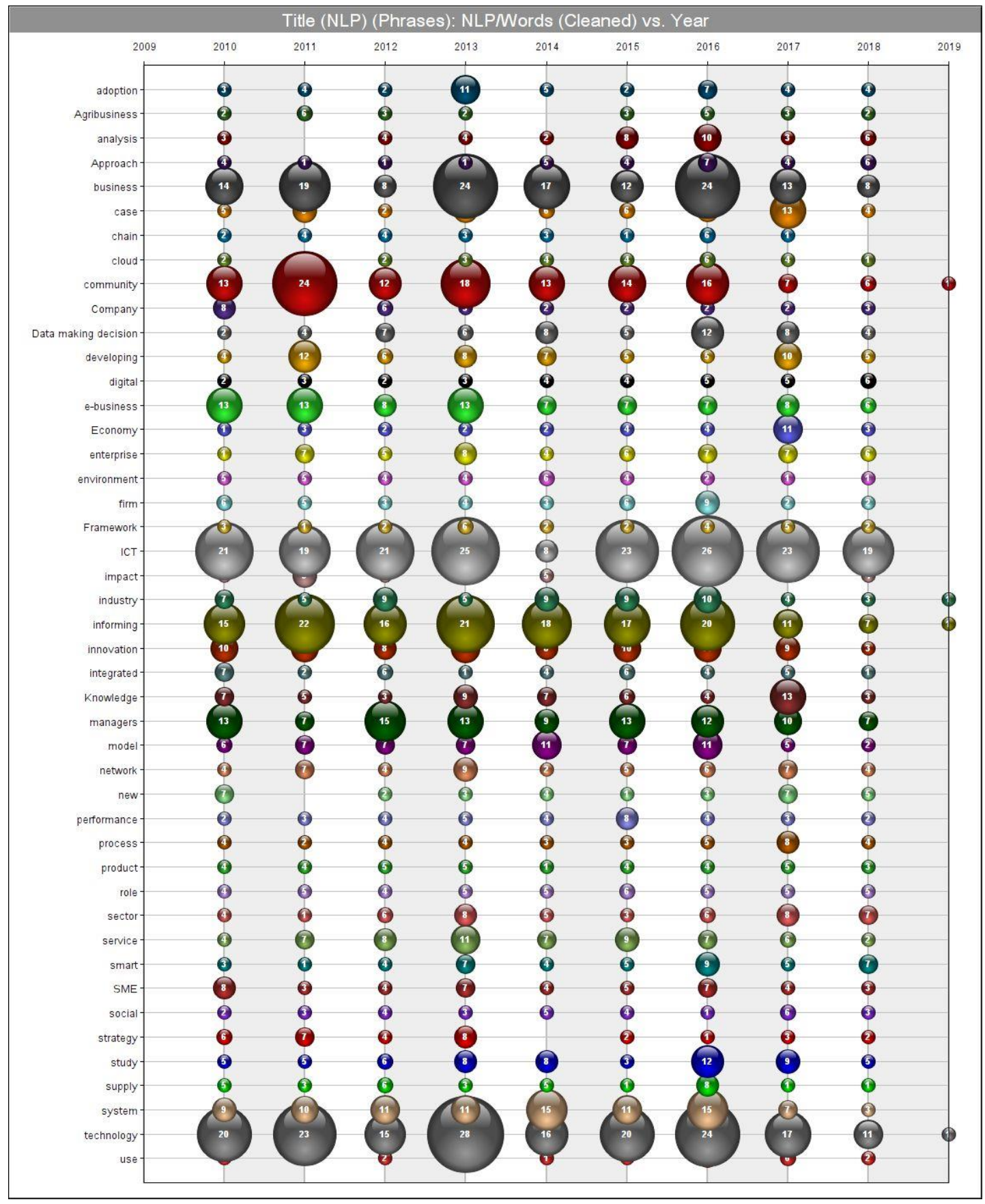

Fig. 1 Gráfico de burbujas por temáticas publicadas

Fuente: Elaborada en Vantagepoint con información de SCOPUS (SCOPUS, 2018) 
Adicionalmente, si se analizan las palabras que más se repinten en las publicaciones se evidencia información interesante como la que muestra el gráfico de aduna, Fig. 2., en donde los círculos de mayor tamaño representan las palabras que más se repiten y la relación de cada término con otro en las publicaciones. Este gráfico además permite encontrar la tendencia que tienen ciertas temáticas a asociarse con otras.

Los bloques de palabras más relevantes son tecnología, seguida por negocios y sistemas que básicamente son los términos que definen la temática de estudio. Los siguientes términos de mayor frecuencia son adopción, análisis, desempeño, estrategia, lo que claramente marca una tendencia que resalta la importancia que sigue teniendo en la literatura científica, el estudio de los procesos de negocio soportados por SI/TI y su relación con las estrategias y el desempeño organizacional, más aún cuando estos términos muestran no solo una fuerte relación entre ellos sino también una relación importante con otros términos como decisiones, éxito, ventajas y perspectivas.

En otro bloque de relaciones encontramos un grupo de palabras como aprendizaje, enseñanza, educación, inteligencia que marca otra clara tendencia en estudios enfocados al uso de TIC en educación.

Otro hallazgo importante que se puede resaltar son las industrias en las que se evidencia aplicación y encontramos la industria de la salud, defensa, educación, agronegocios y bancos, lo que nos lleva a pensar que son los sectores en donde las TIC están siendo un soporte importante en los procesos de análisis y toma de decisiones en este tipo de industrias.

Finalmente resaltar la aparición de temáticas como 4.0, nube, relación hombre-máquina, abierto, automatización, ciudades, móviles, cuidado, salud, crowdsourcing, criptomonedas, mujeres o jóvenes que muestran otras tendencias de trabajos científicos en temáticas diversas, algunas de ellas clásicas, otras emergentes. 


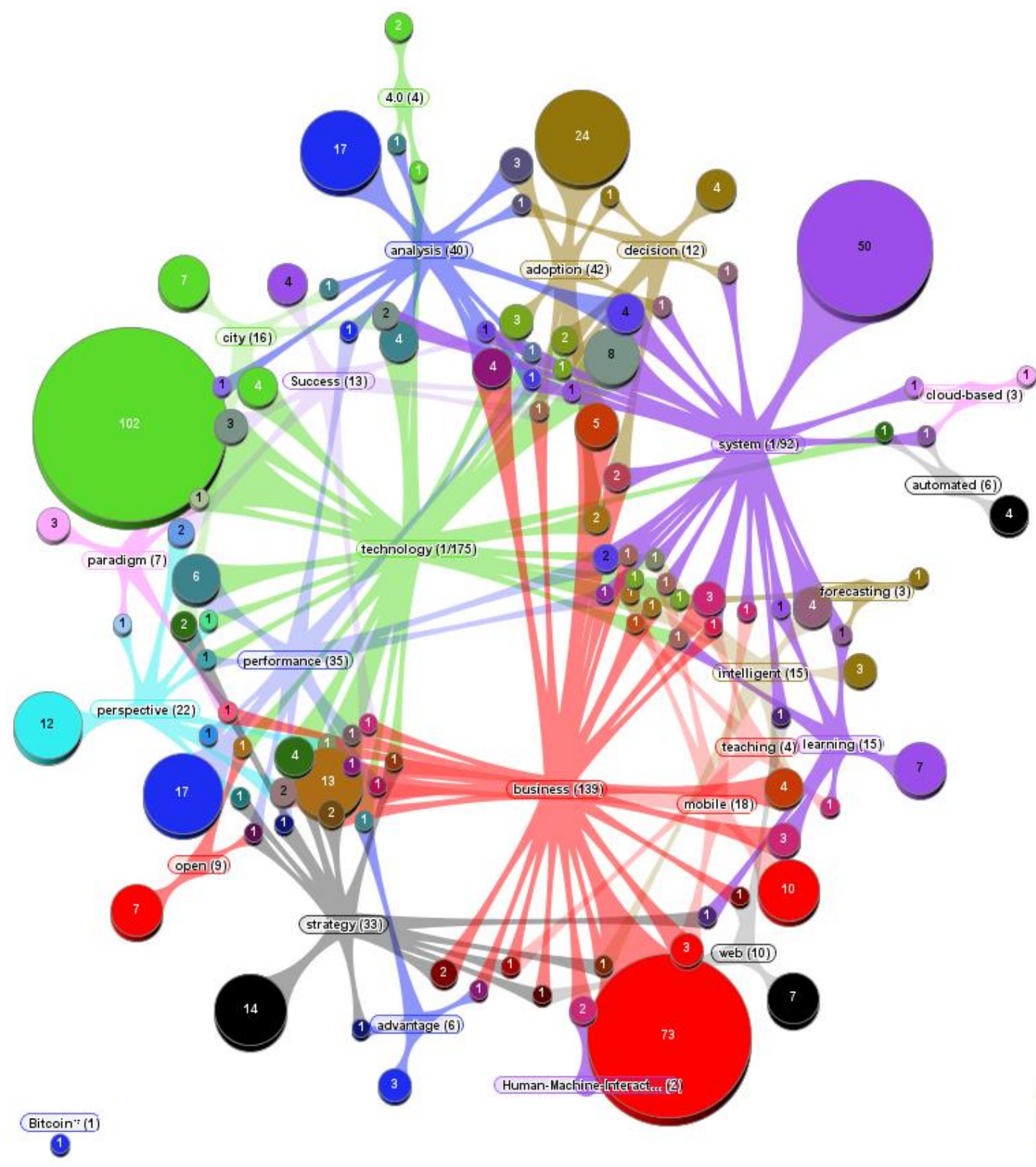

Fig. 2 Gráfico Aduna por términos recurrentes en publicaciones

Fuente: Elaborada en Vantagepoint con información de SCOPUS (SCOPUS, 2018)

(C) $($ ) $९$ Editorial Universitat Politècnica de València 


\section{Análisis del contexto}

\subsection{Análisis externo}

A nivel internacional se destaca el estudio presentado por la Consultora Gartner en el Symposium/ITxpo 2017 en Orlando, Florida, las principales tendencias tecnológicas estratégicas que tendrán mayor impacto y afectarán en la mayoría de las empresas en 2018 son (Gartner, 2017):

- Inteligencia artificial para tomar decisiones incluyendo modelos de «aprendizaje automático»o «machine learning»

- Aplicaciones y análisis inteligentes y sistemas de aprendizaje automático.

- Internet de las Cosas

- La aparición de un gemelo digital que se refiere a la representación digital de una entidad o sistema del mundo real.

- Una «nube» transversal o «edge computing», que es un modelo interconectado que se basa en el procesamiento de información y recopilación de contenido para acercarse en mayor medida con las fuentes de esta información.

- Servicios conversacionales que supondrán un importante cambio de paradigma en cómo los seres humanos interactúan con el mundo digital.

- Una mayor experiencia inmersiva en lo que tiene que ver con realidad virtual (VR) y la realidad aumentada (AR) con potencial beneficio comercial que permitirá a las empresas revisar escenarios específicos de la vida real en los que se pueden aplicar y hacer que los empleados sean más productivos.

- «Blockchain» o «cadena de bloques» integrado en el negocio, que es una tecnología utilizada para la creación de monedas virtuales como el Bitcoin, que además de suponer un cambio de paradigma del sector financiero mundial, puede convertirse en una alternativa disruptiva de los actuales mecanismos centralizados de transacción y mantenimiento de registros, y con ello servir como base de negocios digitales.

De otra parte, de acuerdo al Informe del Foro Económico Mundial titulado "El futuro de los trabajos" se presentan las tendencias y estrategias de la fuerza laboral para Cuarta Revolución Industrial. Este informe indica que entre el rango de roles que experimentarán una demanda creciente en el periodo hasta el año 2022, se encuentran roles como: Analista de datos y científicos, Desarrolladores de software y aplicaciones, y Especialistas en Comercio electrónico y redes sociales que se basan y mejoran significativamente con el uso de la tecnología (World Economic Forum, 2018). Adicionalmente, se espera, según este informe, se evidencia la aceleración de la demanda de una variedad de roles de especialistas relacionados con la comprensión y aprovechamientos de las últimas tecnologías emergentes, estos roles son: especialista en inteligencia artificial y aprendizaje automático, especialista en Big Data, expertos en automatización de procesos, analistas de seguridad de 
la información, experiencia del usuario y Diseñadores de interacción humano-máquina, Ingeniería Robótica y especialistas Blockchain (World Economic Forum, 2018).

Desde un enfoque nacional, se puede destacar el último informe sobre el panorama TIC, elaborado por el Ministerio de Tecnologías de Información y las Comunicaciones (MinTic), que presenta un análisis de la cadena valor de sector TIC en Colombia. Según este informe, teniendo en cuenta el componente de la industria de las plataformas digitales que emerge de la economía digital, la cadena de valor del sector TIC está compuesta por 5 dimensiones: (1) la infraestructura que soporta la utilización de los productos y servicios, (2) la fabricación y/o venta de bienes TIC, (3) la producción de servicios de telecomunicaciones, donde el servicio de Internet comienza a ser el punto de surgimiento de una nueva industria, (4) la industrial de las plataformas digitales; y transversalmente (5) la investigación, desarrollo e innovación necesarias para la continua evolución del sector (PanoramaTIC, 2015). Todos estos elementos de alguna manera impactan las diferentes industrias, razón por la cual deben formar considerarse dentro de las posibilidades de desempeño del futuro profesional.

En este orden de ideas, en el departamento de Santander, en relación al temas de desarrollo económico y su articulación otros instrumentos de desarrollo se plantean estrategias en tema de transporte e infraestructura; tecnologías de la información y las comunicaciones; temas de desarrollo, ciencia e innovación; competitividad y productividad; fomento del desarrollo y empleo; fortalecimiento empresarial e industrial; estrategias de desarrollo turismo; minería e hidrocarburos; gas y energía eléctrica; energía sostenible y alternativa para el desarrollo; cooperación internacional; desarrollo regional; y desarrollo agropecuario y desarrollo rural, entre otros (Gobernación de Santander, 2016). Estas estrategias de han diseñado, teniendo como base, los 17 Objetivos de Desarrollo Sostenible (ODS) y el enfoque de crecimiento verde de la Organización para la Cooperación y el Desarrollo Económico (OCDE) como referente estratégico; y alineado a los objetivos consignados en el Plan Nacional de Desarrollo 2014-2018 (Departamento Nacional de Planeación, 2014).

En lo que tiene que ver con la modernización tecnológica y administrativa se priorizan sectores como Confección, Calzado, Agro-alimentos, Agro-industria y comercio lo que evidencia necesidades regionales que se convierten en oportunidades para el futuro ingeniero industrial, quien va a requerir contar con elementos que le permitan analizar información para apoyar las decisiones de empresas de cualquier sector y optimizar procesos y modelos de negocio (Gobernación de Santander, 2016).

Dentro del Plan de Desarrollo Nacional el objetivo 6 propone el Desarrollo de Infraestructura vial y de transporte e inclusión coherente en las tecnologías de la información y las comunicaciones, así como el acceso a energías sostenibles, en la perspectiva cerrar de brechas productivas. Dentro de sus estrategias se encuentra el reducir 
brechas en acceso a las tecnologías de la información y las comunicaciones en los sitios alejados geográficamente, y garantizar el uso efectivo de las TIC, a través de la conectividad al $100 \%$ de cabeceras municipales que sean centros de influencia indígena (Departamento Nacional de Planeación, 2014).

Por su parte, y en relación a sistemas de información públicos se describe que en materia de tecnologías de la información y las comunicaciones, se impulsará el fortalecimiento y la integración de los sistemas de información con acciones encaminadas a implementar sistemas de planificación financiera, desarrollar la estrategia de datos abiertos -Open Data-, la carpeta ciudadana digital, la estrategia de -Big Data- y la consolidación de la figura de director de Tecnologías y Sistemas de información -Chief Information Officer (CIO)- para las entidades estatales de orden nacional y territorial.

Al mencionar aspectos como acceso a tecnologías y las comunicaciones, y estrategias de datos abiertos, así como estrategias de Big Data, se hace necesario involucrar el concepto de explotación de la información. Esto por esto, que el Consejo Nacional de Política Económica y Social (CONPES ${ }^{2}$ ) desarrolla el documento Conpes 3920, el cual se establece como una política de explotación de datos (Big Data). Este documento tiene por objetivo aumentar el aprovechamiento de datos, mediante el desarrollo de las condiciones para que sean gestionados como activos para generar valor social y económico (CONPES, 2018). Adicionalmente, para materializar este potencial, el documento propone la definición de un marco jurídico e institucional que maximice la obtención de beneficios del aprovechamiento de datos, permita la cooperación entre los sectores público y privado y, al mismo tiempo, refuerce la protección de los derechos de los ciudadanos en el contexto de la creciente transformación de la vida diaria en datos digitales cuantificables y procesables (llamada datificación) (CONPES, 2018).

\subsection{Análisis Institucional}

A nivel Institucional es importante resaltar que la UPB, ha definido 5 focos estratégicos a saber: Energía, TIC, Agua, Alimentación y Territorio, Salud y en central, Humanización y Cultura. Los Focos estratégicos dan cuenta de las principales fortalezas y capacidades internas a nivel de investigación y transferencia, y definen las prioridades temáticas de la universidad, enmarcados en los valores operativos de la institución.

Las líneas orientadoras propuestas por el Foco TIC son (Comité Científico del Foco TIC, 2017): a) Ciencia de datos, b) Ciudades inteligentes, c) Contenidos Digitales, d) Economía

\footnotetext{
2 El Consejo Nacional de Política Económica y Social CONPES es la máxima autoridad nacional de planeación y se desempeña como organismo asesor del Gobierno en todos los aspectos relacionados con el desarrollo económico y social de Colombia.
} 
Digital, e) Infraestructura y conexión, f) Legislación y Regulación, g) Política - Gobierno y ciudadanía en línea, h) Seguridad informática, i) Transformaciones sociales, j) Uso y Apropiación Social de las TIC

Al revisar los focos estratégicos planteados en la UPB para orientar no solo la investigación y transferencia sino también los procesos de docencia y proyección social, se hace evidente los resultados de la revisión de la literatura científica con las líneas definidas por el Foco TIC. Esto sumado a la orientación del plan de Desarrollo de Santander "Santander nos une" (2016 - 2019) ofrece un amplio espectro de posibilidades de acción de la ingeniería industrial desde la perspectiva de las TIC, dado que este Plan de Desarrollo plantea dentro de los temas estratégicos; el desarrollo competitividad y productividad de las empresas de la región usando, entre otras, herramientas tecnológicas de información e infraestructura; que permitan ser un departamento competitivo, frente a la globalización económica (Gobernación de Santander, 2016).

\section{Conclusiones}

A manera de síntesis, se puede identificar un amplio espectro de áreas de trabajo para el programa de Ingeniería Industrial de la UPB BGA, no solo en lo que respecta al contexto regional local que enfrenta retos bien importantes de cara al futuro, sino en el contexto mundial en una industria con tecnologías disruptivas, cambios en los modelos de negocio y de paradigmas.

El estudio permitió identificar competencias emergentes en el ejercicio profesional de los egresados, por lo que el ingeniero industrial del futuro debe estar preparado para desempeñarse en la industria del futuro (en el futuro inmediato se puede hablar de industria 4.0) con nuevos modelos de negocio, en donde uno de los aportes más evidentes se vislumbra en el análisis de grandes cantidades de datos para la toma de decisiones estratégicas y para la gestión de procesos automatizados incluida la inteligencia artificial. Estas tendencias son tan variadas como amplias y si bien podrían abordarse desde las asignaturas optativas, estas solo corresponden a un 5\% de los créditos totales lo que podría ser poco ante la exigencia actual.

Otro cuestionamiento importante que surge al identificar estas tendencias, es la reorientación que está tomando el perfil profesional del ingeniero industrial a la luz de la evolución tecnológica, que de alguna manera exige competencias que no solo entran a complementar otras disciplinas, sino que incluso pueden llegar a solaparse con ellas, lo que invita a repensar de manera radical el programa de ingeniería industrial o incluso a crear nuevos programas académicos más flexibles. Sin embargo, estas decisiones están sujetas a 
contar con infraestructura moderna y docentes formados, recursos con los que el programa actualmente no cuenta ni probablemente podrá contar en el corto plazo.

Finalmente, la invitación es a abordar un análisis mucho más profundo del programa que supera los límites de una transformación curricular tradicional.

\section{Referencias}

Aguilar-Jimenez, A. S. (2010). Análisis del proceso de adopción de tecnologías de información y comunicaciones en actividades de aprovisionamiento empresarial en pequeñas y medianas empresas manufactureras. Una aplicación al sector del mueble en España. Valencia, España: Universidad Politécnica de Valencia.

Carr, N. (2004). Does IT Matter? Information Technology and the Corrosion and Competitive Advantage. Boston, Massachusetts: Harvard Business Press.

Comité Científico del Foco TIC. (2017). Líneas estratégicas del foco TIC.

CONPES. (2018). Política Nacional de Explotación de Datos. Retrieved from www.dnp.gov.co

Davis, G. B. (2000). Information systems conceptual foundations: looking backward and forward. In Organizational and social perspectives on information technology (pp. 61-82). Springer.

Departamento Nacional de Planeación. (2014). Bases del Plan Nacional de Desarrollo. Todos por un nuevo país 2014-2018. Retrieved from www.ccb.org.co

Gartner. (2017). Tedndencias Tecnológicas. In Gartner Symposium/ITxpo 2017. Orlando, Florida. Retrieved from http://www.vailos.com/es/blog/tendencias-tecnologicas-segun-gartner-para-2018/

Gobernación de Santander. (2016). Plan de Desarrollo de Santander "Santander nos une” 20162019. Bucaramanga, Colombia.

Ingeniería Industrial UPB Bucaramanga. (2018). Documento Autoevaluación Programa de Ingeniería Industrial.

Laudon, K., \& Laudon, J. (2007). Sistemas de Información Gerencial. Administración de la Empresa Digital. Pearson.

Ministerio de Educación Nacional. (2018). Resolución 017228 que otorga la Acreditación de Alta Calidad Multicampus a la Universidad Pontificia Bolivariana. Bogotá Colombia.

PanoramaTIC. (2015). Comportamiento macoeconómico del sector TIC en Colombia. Retrieved from www.colombiatic.mintic.gov.co

Pérez González, D. (2005). Contribución de las tecnologías de la información a la generación de valor en las organizaciones: un modelo de análisis y valoración desde la gestión del conocimiento, la productividad y la excelencia en la gestión.

SCOPUS. (2018). Vigilancia TIC-Negocios. 
Universidad Pontificia Bolivariana. (2016). Informe de Autoevaluación Institucional Multicampus.

Universidad Pontificia Bolivariana. (2019a). Transformación Curricular del Programa de Ingeniería Industrial. Bucaramanga, Colombia.

Universidad Pontificia Bolivariana. (2019b). Universidad Pontificia Bolivariana. Retrieved May 15, 2019, from www.upb.edu.co

World Economic Forum. (2018). The Future of Jobs Report 2018. Retrieved from http://www3.weforum.org/docs/WEF_Future_of_Jobs_2018.pdf 